\title{
Experimentally Validated Continuous-time Repetitive Control of Non- minimum Phase Plants with a Prescribed Degree of Stability
}

\author{
Liuping Wang ${ }^{\mathrm{a}}$, Chris T. Freeman ${ }^{\mathrm{b}}$, Eric Rogers ${ }^{\mathrm{b}}$, David H. Owens ${ }^{\mathrm{c}}$ \\ ${ }^{a}$ School of Electrical and Computer Engineering, RMIT University, Victoria 3000 Australia \\ ${ }^{b}$ School of Electronics and Computer Science, University of Southampton, \\ Southampton, SO17 1BJ, UK \\ ${ }^{c}$ Department of Automatic Control and Systems Engineering, University of Sheffield, \\ Sheffield S1 3JD, UK
}

\begin{abstract}
This paper considers the application of continuous-time repetitive control to nonminimum phase plants in a continuous-time model predictive control setting. In particular, it is shown how some critical performance problems associated with repetitive control of such plants can be avoided by use of predictive control with a prescribed degree of stability. The results developed are first illustrated by simulation studies and then through experimental tests on a non-minimum phase electromechanical system.
\end{abstract}

Key words: repetitive control, predictive control, non-minimum phase zeros, experimental evaluation.

\section{Introduction}

Many reference signals encountered in control systems applications are periodic or at least can be accurately approximated by a periodic signal over suitably

Email addresses: liuping.wangermit . edu . au (Liuping Wang), cfeecs.soton.ac.uk (Chris T. Freeman), etar@ecs.soton.ac.uk (Eric Rogers), d.h. owens@sheffield.ac.uk (David H. Owens) 
long time interval. Examples here include those associated with engines, electric motors and generators, converters, and machines performing a repetitive task. Hence it is essential that the output of such a plant has the ability to track a periodic reference signal and/or reject the influence of a periodic disturbance. In the former case, the period will be known a priori by the nature of the task performed, and in the latter it can be identified by using established methods from the areas of signal processing or system identification.

Repetitive control (RC) has emerged as an approach to the general design problem considered in this paper where the basic premise is to use information from previous periods or trials (also termed cycles in some literature) to modify the control signal such that the overall system learns to track a given periodic reference signal. Early references to this approach include [14, 12]. Closely related is iterative learning control (ILC) that is applicable to systems operating in a repetitive manner where the task is to follow a predefined reference trajectory over a specified finite time interval, known as a pass or a trial in the literature, with high precision. In particular, the task is completed and the process is then reset to the starting location for the start of the next pass. The novel principle behind ILC is to suitably use data from previous trials, often in combination with appropriate current trial information, to select the current trial input with the aim of sequentially improving performance. In particular, the aim is to improve performance from trial-to-trial in the sense that the tracking error (the difference between the output on a trial and the specified reference trajectory) is sequentially reduced to either zero (ideal case) or some suitably small value. Overviews of the literature in this area can be found in the survey papers $[3,1]$.

The effect of non-minimum phase zeros on the performance of control schemes is very well known see, for example, [9] and for single-input single-output linear systems is manifest in the form of an undershoot in the initial response to a step 
change in the input (or reference signal closed-loop). Such zeros also introduce performance limitations in RC and ILC for see, for example, $[8,11]$, where the limiting effects on the convergence rate is established. There has been previous work on methods to improve the closed-loop performance in such cases [13, 4], including experimental verification on an electro-mechanical testbed which is where the results developed in this paper are experimentally verified

The new contribution in this paper is a repetitive control algorithm using the predictive control setting developed in [19], and applied in [17], to plants with non-minimum phase zeros. With this new framework, the performance limitation problem caused by the unstable zeros is examined and solved by a simple modification of the cost function. Using a classical result in linear quadratic regulator theory, see, for example, [10] and root-locus analysis it is shown that some of the poles of the repetitive control system move towards the mirrored location of the non-minimum phase zeros of the plant when design is based on minimizing the integral squared errors and the derivatives of the errors between the plant output and the reference signal. This result implies that the convergence rate of a repetitive control system with, for simplicity, one non-minimum phase zero, is dictated by the factor $e^{-\xi t}$, where $\xi$ denotes this zero, as the gain of the system increases. If this non-minimum phase zero is close to the imaginary axis, the convergence rate of the repetitive control system is slow. However, this performance limitation arises from the assumption that the objective function has limited complexity, and the closed-loop root loci are pre-determined. This paper shows that this performance limitation can be overcome if the function to be optimized is generalized to include interactions between the errors, their derivatives, and the state variables. Tuning the resulting repetitive control system for better performance is also addressed.

The presence of interactions in the objective function means that the selection of all the individual elements to achieve a desired effect is a non-trivial task. 
As an alternative, [18] proposed selecting an objective function that results in the repetitive control system with a prescribed degree of stability $\beta>0$, meaning that the closed-loop poles are allocated to the left of the line $s=-\beta$ in the complex $s$-plane, where $s$ denotes the Laplace transform variable. This approach has its origins in the classical method of achieving a prescribed degree of stability in linear optimal control [2] and was adapted to predictive control using real-time optimization in [18]. Moreover, since the new system is developed using the receding horizon control principle, plant operational constraints can, if required, be naturally embedded in its design and implementation.

The next section of this paper summarizes the necessary background on the repetitive-predictive control algorithm and this is followed by use of the rootlocus generated by the repetitive-predictive control system to determine how nonminimum phase zeros affect closed-loop response performance when the traditional error function is used in design. This leads to the introduction of a simple modification of the cost function that overcomes the performance limitations of such zeros, and supporting simulation studies are given. Section 4 reports on the results obtained when the new algorithm is experimentally applied to a nonminimum phase electro-mechanical system. Finally, Section 5 gives an overview of the results in this paper in general terms and discusses possible future research.

\section{The Repetitive-predictive Control Algorithm}

This section gives the essential background to the new results in this paper. In particular, the key steps in the application of the repetitive-predictive control algorithm are described, with a fully detailed treatment in [19]. For notational simplicity, attention is restricted to the single-input single-output (SISO) case. 


\subsection{Embedding the Periodic Disturbance Model}

It is well known from the internal model control principle that in order to reject a periodic disturbance, or follow a periodic reference signal with zero steady-state error, the generator model for the disturbance or the reference must be included in the stable closed-loop control system [5]. In this particular case, the route is to combine the disturbance and plant models to form an augmented model that is then used in analysis and controller design. Suppose, therefore, that the plant to be controlled is described by the state-space model

$$
\begin{aligned}
\dot{x}_{m}(t) & =A_{m} x_{m}(t)+B_{m} u(t)+\Omega_{m} \mu(t) \\
y(t) & =C_{m} x_{m}(t)
\end{aligned}
$$

where $x_{m}(t)$ is the $n_{1} \times 1$ state vector, and $u(t)$ and $y(t)$ the input and output variables respectively (in the case of multiple inputs and/or outputs $u(t)$ and $y(t)$ would be column vectors of compatible dimensions). One way of modeling the input disturbance $\mu(t)$ is as integrated white noise and then the internal model principle is satisfied since the predictive controller has an integrator naturally imbedded in its structure, see [6] and [16] for the discrete and continuous-time cases respectively. A similar route will be followed in this work.

Suppose now that the denominator of the disturbance model transfer-function is of the form

$$
D(s)=s^{\gamma}+d_{1} s^{\gamma-1}+d_{2} s^{\gamma-2}+\ldots+d_{\gamma}
$$

where the roots of this polynomial are either on the imaginary axis or in the open left-half of the complex plane. Also let $\rho$ be the differential operator defined as $\rho f(t)=\frac{d f(t)}{d t}$ and $D(\rho)$ the corresponding polynomial in the denominator of the disturbance model in $\rho$, where $\rho^{k} f(t)=f^{(k)}(t)$ (the $k t h$ derivative of $f(t)$ ). Then it follows immediately that the input disturbance $\mu(t)$ is described by the following 
differential equation

$$
D(\rho) \mu(t)=\left(\rho^{\gamma}+d_{1} \rho^{\gamma-1}+d_{2} \rho^{\gamma-2}+\ldots+d_{\gamma}\right) \mu(t)=0
$$

Define the following auxiliary variables using the disturbance model

$$
\begin{aligned}
z(t) & =D(\rho) x_{m}(t) \\
u_{s}(t) & =D(\rho) u(t)
\end{aligned}
$$

i.e. $z(t)$ and $u_{s}(t)$ are obtained by filtering the state vector $x_{m}(t)$ and the control signal $u(t)$ respectively by the denominator of the transfer-function description of the disturbance model. Also applying the differential operator $D(\rho)$ to both sides of the state equation in the system model (1) gives

$$
D(\rho) \dot{x}_{m}(t)=A_{m} D(\rho) x_{m}(t)+B_{m} D(\rho) u(t)+\Omega_{m} D(\rho) \mu(t)
$$

or

$$
\dot{z}(t)=A_{m} z(t)+B_{m} u_{s}(t)
$$

where the relation $D(\rho) \mu(t)=0$ has been used. Similarly, application of $D(\rho)$ to both sides of (2) gives

$$
D(\rho) y(t)=C_{m} z(t)
$$

or

$$
y^{(\gamma)}(t)=-d_{1} y^{(\gamma-1)}(t)-d_{2} y^{(\gamma-2)}-\ldots-d_{\gamma-1} y^{(1)}(t)-d_{\gamma} y(t)+C_{m} z(t)
$$

where $y^{(n)}$ denotes the $n t h$ derivative of $y$.

Introduce

$$
x(t)=\left[\begin{array}{llllll}
z^{T}(t) & y^{(\gamma-1)}(t) & y^{(\gamma-2)} & \ldots & \dot{y}(t) & y(t)
\end{array}\right]^{T}
$$


and then, using the plant state-space model (1) and (2) and (6) and (7), the statespace model to be used in design is

$$
\begin{aligned}
\dot{x}(t) & =A x(t)+B u_{s}(t) \\
y(t) & =C x(t)
\end{aligned}
$$

where

$$
\begin{gathered}
A=\left[\begin{array}{cccccc}
A_{m} & O & O & \ldots & O & O \\
C_{m} & -d_{1} & -d_{2} & \ldots & -d_{\gamma-1} & -d_{\gamma} \\
O^{T} & 1 & 0 & \ldots & 0 & 0 \\
\ldots & \ddots & & & & \\
O^{T} & 0 & \ldots & 1 & 0 & 0 \\
O^{T} & 0 & \ldots & 0 & 1 & 0
\end{array}\right], B=\left[\begin{array}{c}
B_{m} \\
0 \\
0 \\
\vdots \\
0 \\
0
\end{array}\right] \\
C=\left[\begin{array}{llllll}
0 & 0 & 0 & \ldots & 0 & 1
\end{array}\right]
\end{gathered}
$$

and $O$ denotes the $n_{1} \times 1$ zero vector.

\subsection{The Cost Function and its Minimization}

Predictive control requires the construction of a suitable cost function to be minimized within a moving horizon window and this optimization is performed in real-time, leading to the option of incorporating constraints in the design. The cost function used in predictive control is similar to the classical linear quadratic regulator (LQR) [10], but the solution method is different and is based on numerical minimization of the cost function by directly computing the optimal control trajectory within this moving horizon window. In the discrete-time case, the optimal control trajectory was parameterized by a set of coefficients in conjunction with shift operators [6], and in the continuous-time case the optimal control trajectory was parameterized by a set of continuous-time orthonormal basis functions 
[18]. With this parameterization, the problem of finding the optimal control signal becomes the one of finding the optimal Laguerre coefficients.

Following the continuous-time predictive control case, the filtered control signal $u_{s}(\tau)$ over $0 \leq \tau \leq T_{p}$, where $T_{p}$ is the prediction horizon, is see [18], described in terms of a set of Laguerre functions as

$$
u_{s}(\tau)=L(\tau)^{T} \eta
$$

where $L(\tau)^{T}=\left[\begin{array}{llll}l_{1}(\tau) & l_{2}(\tau) & \ldots & l_{N}(\tau)\end{array}\right]$ and $\eta=\left[\begin{array}{llll}\xi_{1} & \xi_{2} & \ldots & \xi_{N}\end{array}\right]^{T}$. In particular, the set of Laguerre functions are defined explicitly by the following differential equation

$$
\dot{L}(\tau)=A_{p} L(\tau)
$$

where

$$
A_{p}=\left[\begin{array}{cccc}
-p & 0 & \ldots & 0 \\
-2 p & -p & \ldots & 0 \\
\vdots & \vdots & \ddots & 0 \\
-2 p & \ldots & -2 p & -p
\end{array}\right]
$$

and $L(0)$ is the $N \times 1$ vector with each element equal to $\sqrt{2 p}$. Here the parameter $p$ is a scaling factor and $N$ denotes the number of terms used in the orthogonal expansion, and the set of Laguerre functions will have a different response time if the scaling factor $p$ is varied. The key feature of this formulation is that it enables the prediction of future values of the state vector entries as detailed next, where these play a key role in the construction of the cost function on which controller design is based.

Assume that at the current time, say $t_{i}$, the state variable vector $x\left(t_{i}\right)$ is available, but if not then an observer is needed to access the state information through the measurement of input and output signals, which is detailed in Section 2.3. Then 
at the future time $\tau, \tau>0$, the predicted state vector, denoted by $x\left(t_{i}+\tau \mid t_{i}\right)$ is described by the following equation

$$
x\left(t_{i}+\tau \mid t_{i}\right)=e^{A \tau} x\left(t_{i}\right)+\int_{0}^{\tau} e^{A(\tau-\gamma)} B L(\gamma)^{T} d \gamma \eta
$$

Also let $I_{\text {int }}(\tau)$ denote the integral term in this last equation. Then it is routine to show that $I_{i n t}(\tau)$ satisfies the matrix linear differential equation

$$
A I_{\text {int }}(\tau)-I_{\text {int }}(\tau) A_{p}^{T}=-B L^{T}(\tau)+e^{A \tau} B L^{T}(0)
$$

and hence (11) can be written as

$$
x\left(t_{i}+\tau \mid t_{i}\right)=e^{A \tau} x\left(t_{i}\right)+I_{\text {int }}(\tau) \eta
$$

Moreover, the matrix $A_{p}$ is lower triangular and hence (12) has a closed-form solution in the form of a set of linear equations (see $[16,18]$ for more details).

In general terms, the cost function used in predictive control has the form

$$
J=\int_{0}^{T_{p}} x\left(t_{i}+\tau \mid t_{i}\right)^{T} Q x\left(t_{i}+\tau \mid t_{i}\right) d \tau+\eta^{T} R_{L} \eta
$$

where $Q$ and $R_{L}$ are symmetric positive definite and positive semi-definite matrices respectively, written as $Q>0$ and $R_{L} \geq 0$ respectively. For the analysis in this paper, the orthonormal property of the Laguerre functions is used in constructing the cost function by substituting (13) for the predicted state variable vector $x\left(t_{i}+\right.$ $\left.\tau \mid t_{i}\right)$, to give the following form that can then be specialized for problems of practical relevance as illustrated next

$$
\begin{aligned}
J & =\eta^{T}\left\{\int_{0}^{T_{p}} \phi(\tau) Q \phi(\tau)^{T} d \tau+R_{L}\right\} \eta-2 \eta^{T} \int_{0}^{T_{p}} \phi(\tau) Q w\left(\tau \mid t_{i}\right) d \tau \\
& +\int_{0}^{T_{p}} w\left(\tau \mid t_{i}\right)^{T} Q w\left(\tau \mid t_{i}\right) d \tau
\end{aligned}
$$

where $\phi(\tau)=I_{\text {int }}(\tau)$ and $w\left(\tau \mid t_{i}\right)=-e^{A \tau} x\left(t_{i}\right)$. 
Consider the case when a periodic disturbance is to be rejected. Then the control objective in this case is to achieve constant steady-state operation where the plant output (the SISO case is considered) is equal to the desired constant set-point signal and all entries in the state vector $z(t)$ and all derivatives of the plant output are zero.

In the case when set-point following of a periodic signal, say $r(t)$, is required it is necessary to assume that the set-point signal is differentiable up to the order of $\gamma-1$ where $\gamma$ is the order of the disturbance model used in the design. The associated cost function for set-point following is often chosen as

$$
J=\int_{0}^{T_{p}} e\left(t_{i}+\tau \mid t_{i}\right)^{T} e\left(t_{i}+\tau \mid t_{i}\right) d \tau+\int_{0}^{T_{p}} u_{s}(\tau)^{T} R u_{s}(\tau) d \tau
$$

where

$$
e\left(t_{i}+\tau \mid t_{i}\right)=\left[\begin{array}{c}
r^{(\gamma-1)}\left(t_{i}\right)-y^{(\gamma-1)}\left(t_{i}+\tau \mid t_{i}\right) \\
\vdots \\
r^{(1)}\left(t_{i}\right)-y^{(1)}\left(t_{i}+\tau \mid t_{i}\right) \\
r\left(t_{i}\right)-y\left(t_{i}+\tau \mid t_{i}\right)
\end{array}\right]
$$

Let $C_{2}$ be the $\gamma \times\left(n_{1}+\gamma\right)$ matrix defined by

$$
C_{2}=\left[\begin{array}{ll}
o_{n 1} & I_{\gamma}
\end{array}\right]
$$

where $o_{n 1}$ is the $\gamma \times n_{1}$ zero matrix and $I_{\gamma}$ is the $\gamma \times \gamma$ identity matrix. Then it is shown in [19] that the cost function (16) is identical to that of (14) with $Q=C_{2}^{T} C_{2}$. Also, since $r(t)$ is periodic, the vectors, $\phi(\tau)$ and $w\left(\tau \mid t_{i}\right)$ take the form

$$
\phi(\tau)=C_{2} I_{\text {int }}(\tau)
$$

and

$$
w\left(\tau \mid t_{i}\right)=\left[\begin{array}{c}
r^{(\gamma-1)}\left(t_{i}\right) \\
\vdots \\
r\left(t_{i}\right)
\end{array}\right]-C_{2} e^{A \tau} x\left(t_{i}\right)
$$


respectively.

Consider the unconstrained minimization with respect to the parameter vector $\eta$ of the general cost function (15) in the absence of hard constraints. Then the minimizing $\eta$ is the least squares solution

$$
\eta=\left\{\int_{0}^{T_{p}} \phi(\tau) Q \phi(\tau)^{T} d \tau+R_{L}\right\}^{-1} \int_{0}^{T_{p}} \phi(\tau) Q w\left(\tau \mid t_{i}\right) d \tau
$$

where the optimal $\eta$ and control $u_{s}(\tau), 0 \leq \tau \leq T_{p}$, can be expressed in terms of the associated Laguerre functions as

$$
u_{s}(\tau)=L(\tau)^{T} \eta
$$

Also, by the principle of receding horizon control, the optimal control $u_{s}(t)$ for the unconstrained problem at time $t_{i}$ is

$$
u_{s}\left(t_{i}\right)=L(0)^{T} \eta
$$

In this design, the Laguerre scaling parameter $p$ and the number of terms used, $N$, are the performance tuning parameters. When $N$ is large, with a long prediction horizon $T_{p}$, the filtered control trajectory $u_{s}($.$) will closely match the underlying$ optimal control trajectory defined by the linear quadratic regulator (LQR) [18].

Having computed $u_{s}(t)$ at time $t_{i}$, the final step is to convert this into the actual control signal $u(t)$ to be applied at $t_{i}$. The procedure for this is detailed next.

\subsection{Control Implementation}

Given the optimal $u_{s}(t)$ at time $t_{i}$, the actual control signal $u(t)$ at this time satisfies $u_{s}(t)=D(\rho) u(t)$. Also introduce

$$
U(t)=\left[\begin{array}{lllll}
u^{(\gamma-1)}(t) & u^{(\gamma-2)}(t) & \ldots & u^{(1)}(t) & u(t)
\end{array}\right]^{T}
$$

and using a controllable canonical form realization gives

$$
\dot{U}(t)=A_{u} U(t)+B_{u} u_{s}(t)
$$


where

$$
A_{u}=\left[\begin{array}{ccccc}
-d_{1} & -d_{2} & \ldots & -d_{\gamma-1} & -d_{\gamma} \\
0 & 1 & \ldots & 0 & 0 \\
\ldots & \ddots & & & \\
0 & \ldots & 1 & 0 & 0 \\
0 & 0 & \ldots & 1 & 0
\end{array}\right], B_{u}=\left[\begin{array}{c}
1 \\
0 \\
\vdots \\
0 \\
0
\end{array}\right]
$$

Moreover, approximating the differential equation (22) with a sampling interval of $\Delta t$, gives the optimal control at $t_{i}$ as

$$
U\left(t_{i}\right)=\left(I-A_{u} \Delta t\right)^{-1} U\left(t_{i-1}\right)+\left(I-A_{u} \Delta t\right)^{-1} B_{u} u_{s}\left(t_{i}\right) \Delta t
$$

where the backward difference approximation, $\left.\frac{d f(t)}{d t}\right|_{t=t_{i}} \approx \frac{f\left(t_{i}\right)-f\left(t_{i-1}\right)}{\Delta t}$, is used. The actual control $u\left(t_{i}\right)$ is computed using the optimal signal $u_{s}\left(t_{i}\right)$ and the previous states of the control derivatives and the control itself.

In this formulation the computation of the actual control vector is iterative. At the instant when the control system is switched on, the initial conditions of the control vector are specified, i.e., $U\left(t_{0}\right)$, and these can be chosen to correspond to the actual plant control states. For example, the control signal $u\left(t_{0}\right)$ can be taken as the actual input to the plant with the derivatives of $u(t)$ equal to zero. With this selection, the recursive computation will automatically update the actual control signal to the plant, and the implementation of the control system is performed without additional information such as the steady-state value of the plant output.

The resulting state-space model here contains the auxiliary state variable vector entries $z(t), y(t)^{(\gamma-1)}, \ldots, y^{(1)}(t)$ and $y(t)$ and hence derivatives of the entries in the original state variable vector $x_{m}(t)$ and the plant output $y(t)$. Moreover, it is not desirable to implement the continuous-time predictive controller using $z(t)$ even if $x_{m}(t)$ is available because differentiation of a signal will amplify any 
existing measurement noise. Instead, an observer is recommended to estimate the augmented state vector $x(t)$, even in the case when all entries of the original state vector $x_{m}(t)$ are available from measurements. The observer structure is chosen to match the augmented model and has the form

$$
\frac{d \hat{x}(t)}{d t}=A \hat{x}(t)+B u_{s}(t)+K_{o b}(y(t)-\hat{y}(t))
$$

where $\hat{x}(t)$ and $\hat{y}(t)$ are the estimated state vector and output respectively, and $K_{o b}$ is the observer gain matrix.

\section{Repetitive-predictive Control with a Prescribed Degree of Stability}

This section investigates issues associated with tuning the performance of the repetitive-predictive controller detailed in the previous section, starting with a cost function used in predictive control, and leading on to analysis that shows the limitation in closed-loop control performance that would result if this class of cost function were to be used in repetitive predictive control of a non-minimum phase plant. The outcome is control law design with a prescribed degree of stability to achieve desired closed-loop performance when applied to such plants.

The first case considered is the effect of high gain control with the following cost function commonly used, see for example [6], in predictive control of SISO plants (this is a special case of (16) where the derivatives of the error signal are not included)

$$
J=\int_{0}^{T_{p}}\left(r\left(t_{i}\right)-y\left(t_{i}+\tau \mid t_{i}\right)\right)^{2} d \tau+R \int_{0}^{T_{p}} u_{s}(\tau)^{2} d \tau
$$

In the absence of constraints, it is known [18] that if exponential data weighting is employed then the predictive controller converges to the corresponding linear quadratic regulator with sufficiently large prediction horizon $T_{p}$ and large $N$ 
(where this last parameter is the number of terms included in the orthonormal expansion). Therefore, the closed-loop poles of the predictive repetitive controller will follow the stable branches of the dual root-locus, dictated by the choice of the weight coefficient $R$ [10]. Also the optimal control is the filtered control signal $u_{s}(t)$, and hence a transfer-function describing the relationship between the output and the control signal is required for root-locus analysis. By following the procedure given in [18], the required transfer-function is

$$
G_{s}(s)=\frac{Y(s)}{U_{s}(s)}=\frac{1}{D(s)} G(s)
$$

where $D(s)$ is the denominator polynomial of the disturbance model transferfunction and $G(s)=C_{m}\left(s I_{n_{1}}-A_{m}\right)^{-1} B_{m}$ is the plant transfer-function.

The closed-loop poles of the repetitive-predictive control system are determined by the stable zeros of

$$
1+\frac{1}{R} \frac{G(s) G(-s)}{D(s) D(-s)}=0
$$

and, from root-locus analysis, it follows that a branch (or branches) generated by the closed-loop poles will converge to the mirrored location in the complex plane of the non-minimum phase zero (or zeros) of the plant transfer-function. If these zeros are close to the imaginary axis then the closed-loop response speed is limited as these zeros become the closed-loop poles as the weighting on the control in the cost function $(R)$ is reduced. This is also true if the plant has zeros with negative real parts but close to the imaginary axis of the complex plane.

As an example, consider the case when

$$
G(s)=\frac{s-1}{(s+3)(s+6)}
$$

and the denominator of the transfer-function describing the disturbance model has the form

$$
D(s)=s\left(s^{2}+w_{1}^{2}\right)
$$


where $w_{1}=\frac{2 \pi}{3}$. Suppose also the repetitive predictive control system is required to follow a set-point signal consisting of a sinusoidal signal with a period of 3 seconds and a constant bias. For a given weighting $R$ in the cost function (25), the closed-loop poles are given by the stable solutions of

$$
1+\frac{1}{R} \frac{(-s-1)(s-1)}{(-s+3)(s+3)(-s+6)(s+6)(-s)(s)\left(s^{2}+w_{1}^{2}\right)^{2}}=0
$$

and varying $R$ from 0 to $\infty$, produces the dual root-locus shown in Figure 1. In this

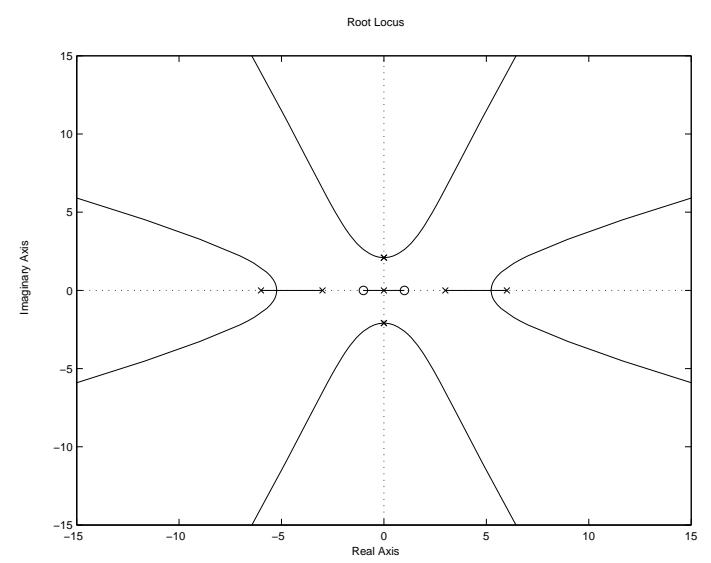

Figure 1: Dual root-locus generated by (11) and (29) with $w=0$ and $w_{1}=\frac{2 \pi}{3}$

case the dominant pole moves from the origin to -1 , which is the mirror location of the non-minimum zero.

Consider now the case when $D(s)$ includes one more periodic mode and takes the form

$$
D(s)=s\left(s^{2}+w_{1}^{2}\right)\left(s^{2}+w_{2}^{2}\right)
$$

where $w_{1}=\frac{2 \pi}{3}$ and $w_{2}=\frac{4 \pi}{3}$, resulting in the dual root-locus of Figure 2. Clearly, the additional periodic mode adds four branches in the dual root-locus but that for the dominant pole remains unchanged.

This example highlights the potential performance limitations when controlling a non-minimum phase plant by repetitive predictive control if a traditional cost 


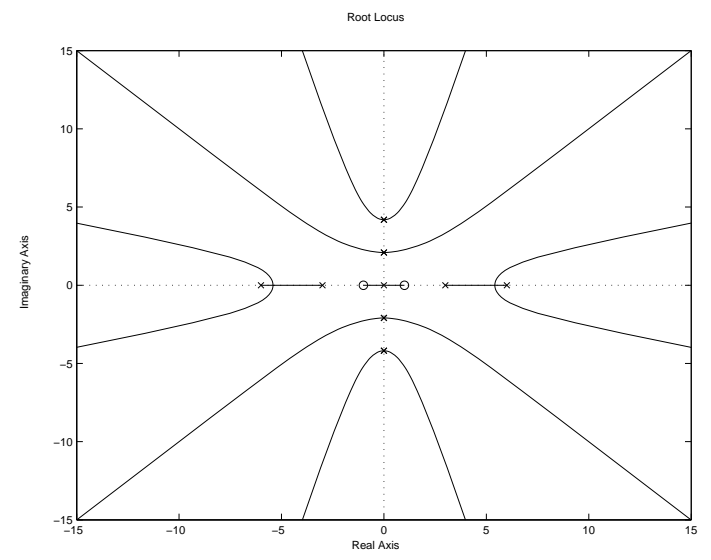

Figure 2: Dual root-locus generated by (11) and (29) with $w=0, w_{1}=\frac{2 \pi}{3}, w_{2}=\frac{4 \pi}{3}$

function is used in the design, and there has been some work on this problem reported in the repetitive control literature. Next predictive repetitive control with a prescribed degree of stability is developed as a solution to this problem.

\subsection{Predictive Repetitive Control with a Prescribed Degree of Stability}

The analysis here is in a linear quadratic regulator setting where the general form of the cost function for the repetitive-predictive control system is

$$
J=\int_{0}^{T_{p}}\left[x\left(t_{i}+\tau \mid t_{i}\right)^{T} Q x\left(t_{i}+\tau \mid t_{i}\right)+u_{s}(\tau)^{T} R u_{s}(\tau)\right] d \tau
$$

where $Q \geq 0, R>0$, and $x(\cdot)$ is the augmented state vector that includes $x_{s}(\cdot)$ and the output $y(\cdot)$ and its derivatives. Moreover, with this general form cost function, the resulting closed-loop poles of the repetitive-predictive control system will not necessarily obey the root-locus rule given above.

The matrix $Q$ is of dimension $\left(n_{1}+\gamma\right) \times\left(n_{1}+\gamma\right)$ and it is very difficult and time consuming to select the individual elements in $Q$ to achieve desired closedloop performance. In addition, the formulation of the predictive repetitive control problem has led to an augmented system state matrix $(A)$ that has $\gamma$ poles on the 
imaginary axis of the complex plane. As a result, the predictive repetitive control system is numerically ill-conditioned and there is a need to improve the numerical conditioning and develop a systematic way to tune its closed-loop performance.

One approach to predictive control with a prescribed degree of stability has been developed in [18] where the resulting design also overcomes the numerical ill-conditioning problem. In particular, the eigenvalues of the state matrix $A$ in the augmented state-space model (9) are determined, where if the plant is stable the unstable eigenvalues of $A$ come from the periodic modes that have been embedded in the model. It is, however, essential to use a stable model in the predictive computation and the strategy here is to select an exponential weighting $\alpha$ and $A-\alpha I$ in this computation, where $\alpha>0$ if the plant is stable. If the plant is unstable with all its eigenvalues lying to the left of the line $s=\epsilon$ line in the complex plane, where $\epsilon>0$, then $\alpha>\epsilon$ is required.

Once the exponential weighting factor $\alpha$ is selected, the eigenvalues of the matrix $A-\alpha I$ are fixed. Since this matrix is stable for an appropriate choice of $\alpha$, the prediction of the state variables is numerically well conditioned and the prediction horizon $T_{p}$ is selected sufficiently large to capture the transformed state variable response. In general, if the eigenvalues of $A-\alpha I$ were further away from the imaginary axis in the complex plane, then a smaller $T_{p}$ can be used.

The use of exponential data weighting alters the original closed-loop performance as specified by the cost function weighting matrices $Q$ and $R$, and in order to compensate for this variation the $Q$ matrix is replaced by

$$
Q_{\alpha}=Q+2 \alpha P
$$

where $P$ is the solution of the Riccati equation

$$
P A+A^{T} P-P B R^{-1} B^{T} P+Q=0
$$


To achieve a prescribed degree of stability, $\beta$, the $P$ matrix is found as the solution of the Riccati equation

$$
P(A+\beta I)+(A+\beta I)^{T} P-P B R^{-1} B^{T} P+Q=0
$$

and $Q_{\alpha}$ from

$$
Q_{\alpha}=Q+2(\alpha+\beta) P
$$

As a numerical example, consider the case when

$$
G(s)=\frac{s-0.1}{(s+3)(s+6)}
$$

which has a non-minimum phase zero at 0.1 with the periodic modes $w_{0}=0$ and $w_{1}=2 \pi / 3$ embedded in the design. We choose the exponential weighting factor as $\alpha=0.18$ to ensure numerical stability in the computation. In this case the dominant closed-loop pole will converge to -0.1 , as the weighting $R$ is reduced. As an illustration of this consider the choice $R=10^{-8}, p=0.1$ and $N=15$, with resulting closed-loop poles at

$$
5.9971, \quad-3.1095-0.8339 \pm 1.2777 j, \quad-0.1
$$

Figure 3 shows the corresponding closed-loop response where the very slow convergence rate in tracking the periodic reference signal is evident. To improve the convergence rate, the slow dominant closed-loop pole needs to be removed by using prescribed degree of stability design, where here the requirement is that all closed-loop poles must be to the left of the line $\beta=-2$ in the complex plane. Also $Q$ is unaltered but $R=1$ is used (instead of a very small number) for better numerical conditioning

The Laguerre scaling factor $p=2$ is applied to the guessed dominant closedloop pole in this case $(\beta), P$ is obtained by solving the Riccati equation (35) and 


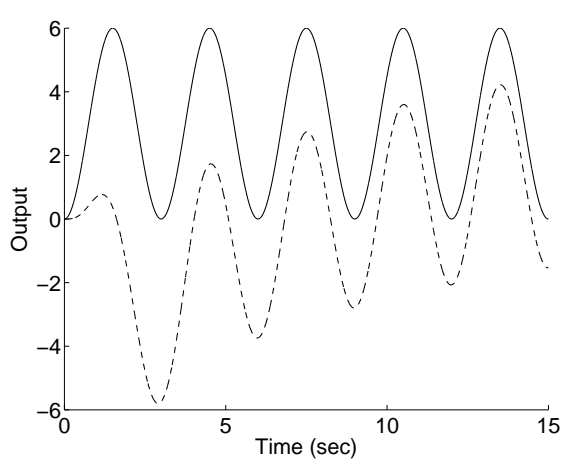

(a) Output and Reference

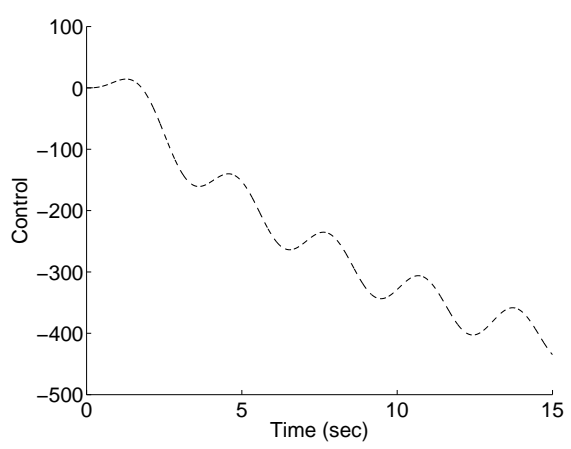

(b) Control signal

Figure 3: Repetitive-predictive control without prescribed degree of stability.

$Q_{\alpha}$ from (36). With this design, the predictive repetitive control system has the closed-loop poles

$$
-6, \quad-4.0138 \pm 2.1491 j, \quad-3.9155, \quad-3.0005
$$

The closed-loop response with the prescribed degree of stability design in place is shown in Figure 4. For comparative purposes, the closed-loop response without the prescribed degree of stability is also shown and is clearly not tracking the periodic reference signal.

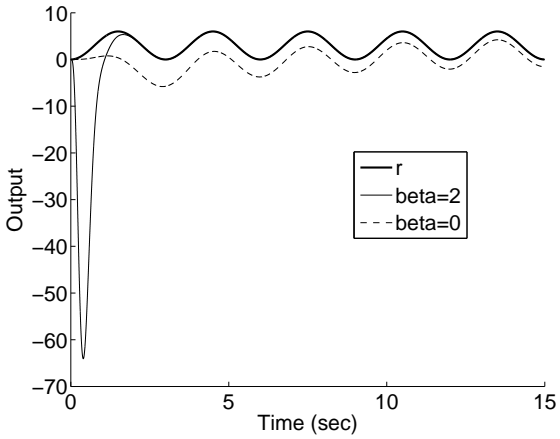

(a) Output and Reference

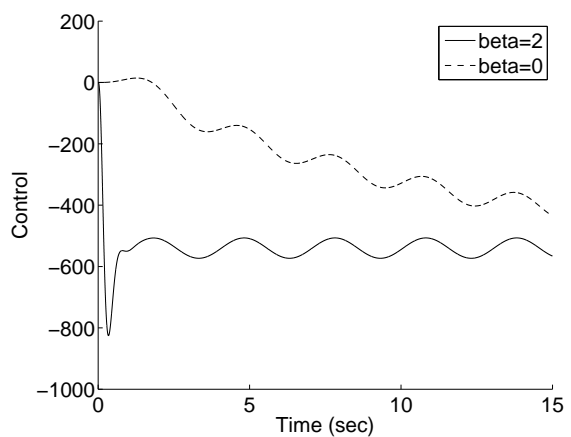

(b) Control signal

Figure 4: Repetitive-predictive control with and without prescribed degree of stability. 


\section{Experimental Results}

The test facility used to provide the experimental results is shown in Figure 5 , and has previously been used to evaluate a number of RC and ILC algorithms (see $[7,15])$ for details). It has been specifically designed to produce the non-

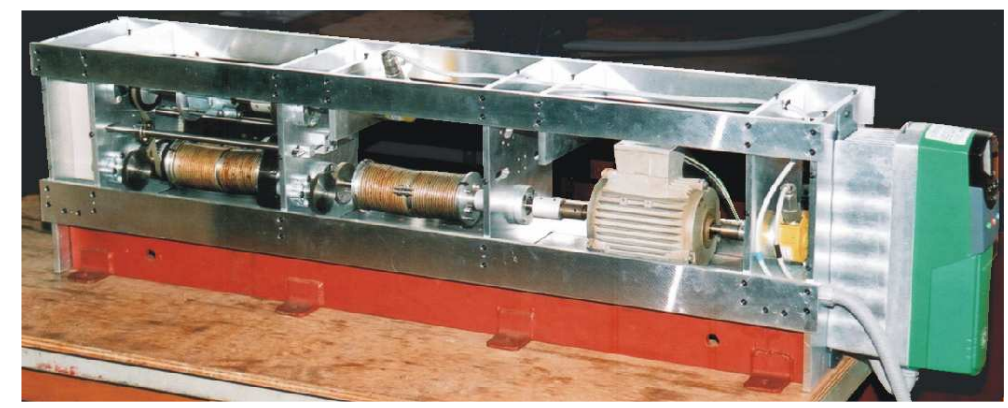

Figure 5: Experimental test facility.

minimum phase characteristic, and consists of a rotary mechanical system of inertias, dampers, torsional springs, a timing belt, pulleys and gears. A 1000 pulse/rev encoder records the output shaft position and a standard squirrel cage induction motor supplied by an inverter, operating in Variable Voltage Variable Frequency (VVVF) mode, drives the load. The system input is the voltage supplied to the inverter and the system output is in radians. The system can be represented using the continuous time plant transfer function

$$
G(s)=\frac{1.202(4-s)}{s(s+9)\left(s^{2}+12 s+56.25\right)}
$$

which has been identified in previous work. A sampling period of $0.0001 \mathrm{~s}$ has been used in the experimental tests performed. As in the last section, values of $\alpha=0.18$ and $N=15$ have been selected, and the periodic modes $w_{0}=0$ and $w_{1}=2 \pi / 3$ have also been embedded. The same $Q$ and $R$ values are also used and $p$ is set equal to $\beta$. Figure 6 shows the experimental tracking results and corresponding 
input using $\beta=0.8$. Here the closed-loop poles are

$$
-9.0000, \quad-6.0000 \pm 4.5000 j, \quad-1.5929 \pm 2.1103 j, \quad-1.6000 \pm 0.0007 j
$$

Figure 7 shows results using $\beta=1.8$, and the increase in convergence speed is

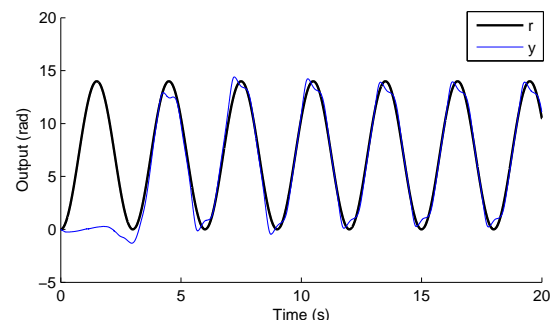

(a) Output and Reference

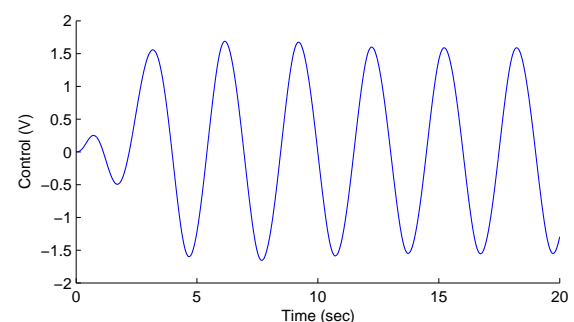

(b) Control signal

Figure 6: Experimental results using beta $=0.8$.

clearly evident. Here the closed loop poles are

$$
-9.0000, \quad-6.0000 \pm 4.5000 j, \quad-3.6002 \pm 2.0946 j, \quad-3.6161, \quad-3.5832
$$

A further increase in convergence rate is observed when $\beta=3.8$, as shown in

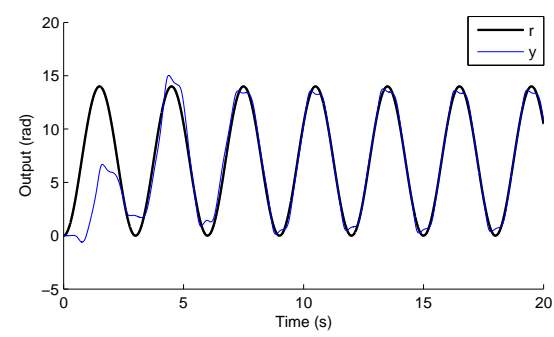

(a) Output and Reference

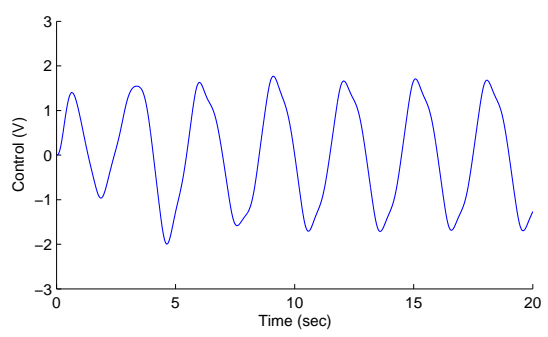

(b) Control signal

Figure 7: Experimental results using beta $=1.8$.

Figure 8. In this case the closed loop poles are

$$
-6.0000 \pm 4.5000 j, \quad-7.6018 \pm 2.0940 j, \quad-9.0000, \quad-7.6754, \quad-7.5188
$$




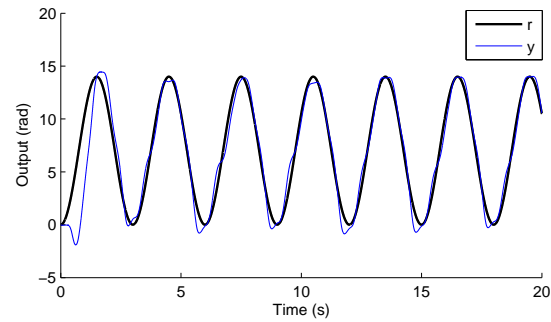

(a) Output and Reference

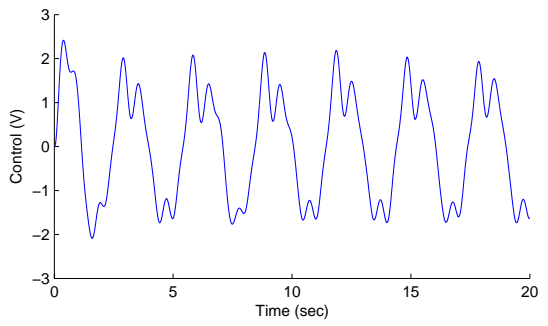

(b) Control signal

Figure 8: Experimental results using beta $=3.8$.

If beta is increased excessively, the controller robustness suffers and performance is degraded, as confirmed by Figure 9 in which a value of $\beta=4.2$ has been used, and the closed loop poles are

$$
-6.0000 \pm 4.5000 j, \quad-8.4027 \pm 2.0943 j, \quad-9.0000, \quad-8.4923, \quad-8.2998
$$

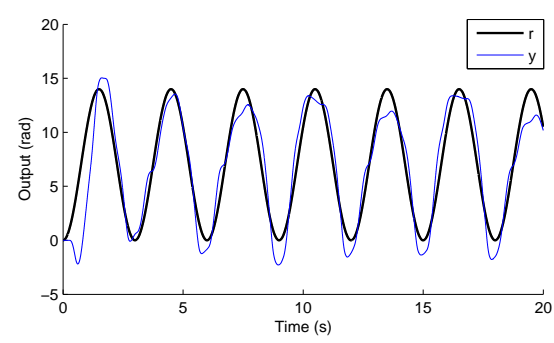

(a) Output and Reference

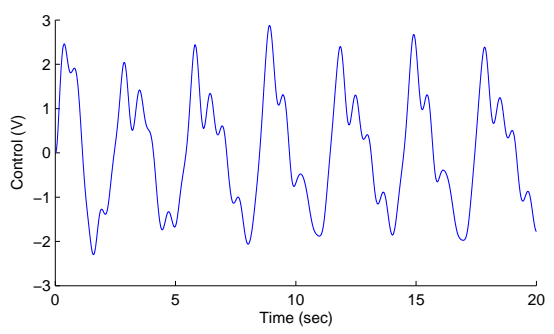

(b) Control signal

Figure 9: Experimental results using beta $=4.2$.

\section{Conclusions}

In this paper an approach is developed to disturbance rejection and set-point following of periodic signals in the framework of predictive control with constraints. The predictive control system is designed with embedded periodic compo- 
nents in the augmented model where a set of continuous-time Laguerre functions is used to describe the inversely filtered control signal. As a result, the control system tracks (or rejects) periodic signals with zero steady state errors. Illustrative simulation studies have been reported and the designs experimentally verified on a non-minimum phase electro-mechanical testbed with excellent agreement between simulated and measured performance. One obvious area for further work is to include control constraints.

Acknowledgment. The work reported here was partially undertaken whilst Professor Eric Rogers was a Visiting Professor at RMIT University, Melbourne supported by the Australian Advanced Manufacturing Cooperative Research Center (AMCRC).

\section{References}

[1] H.-S. Ahn, Y. Chen, and K. L. Moore. Iterative learning control: Brief survey and categorization. IEEE Transactions on Systems, Man, and Cybernetics Part C: Applications and Reviews, 37(6):1099-1121, 2007.

[2] B. D. O. Anderson and J. B. Moore. Optimal Control Linear Quadratic Methods. Prentice-Hall, 1989.

[3] D. A. Bristow, M. Tharayil, and A. G. Alleyne. A survey of iterative learning control. IEEE Control Systems Magazine, 26(3):96-114, June 2006.

[4] Z. Cai, C. T. Freeman, P. L. Lewin, and E. Rogers. Iterative learning control for a non-minimum phase plant based on a reference shift algorithm. Control Engineering Practice, 16:633-643, 2008. 
[5] B. A. Francis and W. Wonham, The internal model principle of control theory. Automatica, 12(5):457-465, 1976.

[6] D. W. Clarke, C. Mohtadi, and P. S. Tuffs. Generalized predictive control Part 1: the basic algorithm. part 2: Extensions and interpretations. Automatica, 23:137-160, 1987.

[7] C. T. Freeman, P. L. Lewin, and E. Rogers. Further results on the experimental evaluation of iterative learning control algorithms for non-minimum phase plants. International Journal of Control, 80(4):569-582, 2007.

[8] C. T. Freeman, P. L. Lewin, E. Rogers, D. H. Owens, and J. Hatonen. An optimality based repetitive control algorithm for discrete-time systems. IEEE Transactions on Circuits and Systems I: Fundamental Theory and Applications, 55(1):412-423, 2008.

[9] G. C. Goodwin, S. F. Graebe, and M. E. Salgado. Control System Design. Prentice-Hall, 2000.

[10] T. Kailath. Linear Systems. Prentice-Hall, 1980.

[11] D. H. Owens, B. Chu, E. Rogers, C. T. Freeman, and P. L. Lewin. Modeling the influence of non-minimum phase zeros on gradient based linear iterative learning control. Systems and Control Letters, 2009. submitted.

[12] M. Nakando S. Hara, Y. Yamamoto T. Omata. Repetitive control system: a new type of servo system for periodic exogenous signals. IEEE Transactions on Automatic Control, 33(7):659-668, 1988.

[13] M. Steinbuch. Repetitive control for systems with uncertain period-time. Automatica, 38(12):2103-2109, 2002. 
[14] S. Iwai T. Inoue, and M. Nakano. High accuracy control of a proton synchrotron magnetic power supply. Proceedings of 8th IFAC World Congress, pages 3137-3142, 1981.

[15] C. T.Freeman, P. L. Lewin, E. Rogers, D. H. Owens, and J. J. Hatonen. An optimality based repetitive control algorithm for discrete-time systems. IEEE Transactions on Circuits and Systems I: Fundamental Theory and Applications, 55(1):412-423, 2008.

[16] L. Wang. Continuous time model predictive control using orthonormal functions. International Journal of Control, 74:1588-1600, 2001.

[17] L. Wang, S. Smith, and C. Chessari. Continuous-time model predictive control of food extruder, Control Engineering Practice, 16: 1173-1183, 2008.

[18] L. Wang. Model Predictive Control System Design and Implementation using MATLAB. Springer-Verlag, 2009.

[19] L. Wang, P. Gawthorp, D. H. Owens, and E. Rogers. Switched linear model predictive controllers for periodic exogenous signals. International Journal of Control, 2010. In press. 